\title{
Organics in cometary and interplanetary dust
}

\author{
A. Chantal Levasseur-Regourd ${ }^{1}$ and Jeremie Lasue ${ }^{2}$ \\ ${ }^{1}$ Aeronomie IPSL, Univ. Pierre et Marie Curie, Paris, France \\ email: aclr@aerov.jussieu.fr \\ ${ }^{2}$ Aeronomie IPSL and LPG
}

\begin{abstract}
While gaseous carbon-rich species in cometary comae (coming from the nuclei icy component) are extensively studied by spectroscopic remote observations, so-called CHONs dust particles, i. e. organic compounds coming from the nuclei refractory component, have mostly been studied by dust mass spectrometers flying through the comae of comets $1 \mathrm{P} /$ Halley and $81 \mathrm{P} /$ Wild 2. However, remote observations of the light scattered by dust in cometary comae and in the interplanetary medium, coupled with both numerical and experimental simulations, have recently allowed us to confirm that such particles harbor a significant fraction of absorbing material, presumably consisting of organic compounds (Levasseur-Regourd et al. PSS 2007, Lasue et al. A\&A 2007).

We estimate the fraction of absorbing material present in cometary dust for extensively observed comets (e.g., 1P/Halley, C/1995 O1 Hale-Bopp) and in the interplanetary dust (from zodiacal light observations). We also establish that, besides compact particles, fluffy aggregates are definitely present in these media. The properties (e.g., size distribution, morphology, composition) of the cometary and interplanetary dust particles, as inferred from light scattering data analysis, are compared with those of the IDPS collected in the upper Earth atmosphere and of the unique samples returned by the Stardust mission at Wild 2. The results are discussed in terms of the formation of comets in the protosolar nebula, and of the possible survival, at the epoch of late early bombardment, of cometary organics embedded in fluffy aggregates.
\end{abstract}

\section{Discussion}

ZINNER: What can you say about the variations between different comets in terms of your measurements?

LEVASSEUR-REgOURD: It's something I did not address here. We have mainly been making comparisons between Halley dust and Hale-Bopp dust. Using light scattering measurements we find that that Hale-Bopp dust has larger amounts of aggregates and tiny grains. Also the drop off in Comet Halley dust as a function of the distance from the nucleus was definitely different for main coma dust particles and dust particles that were observed in the jets, particles which are most likely coming from the subsurface of the nucleus. 


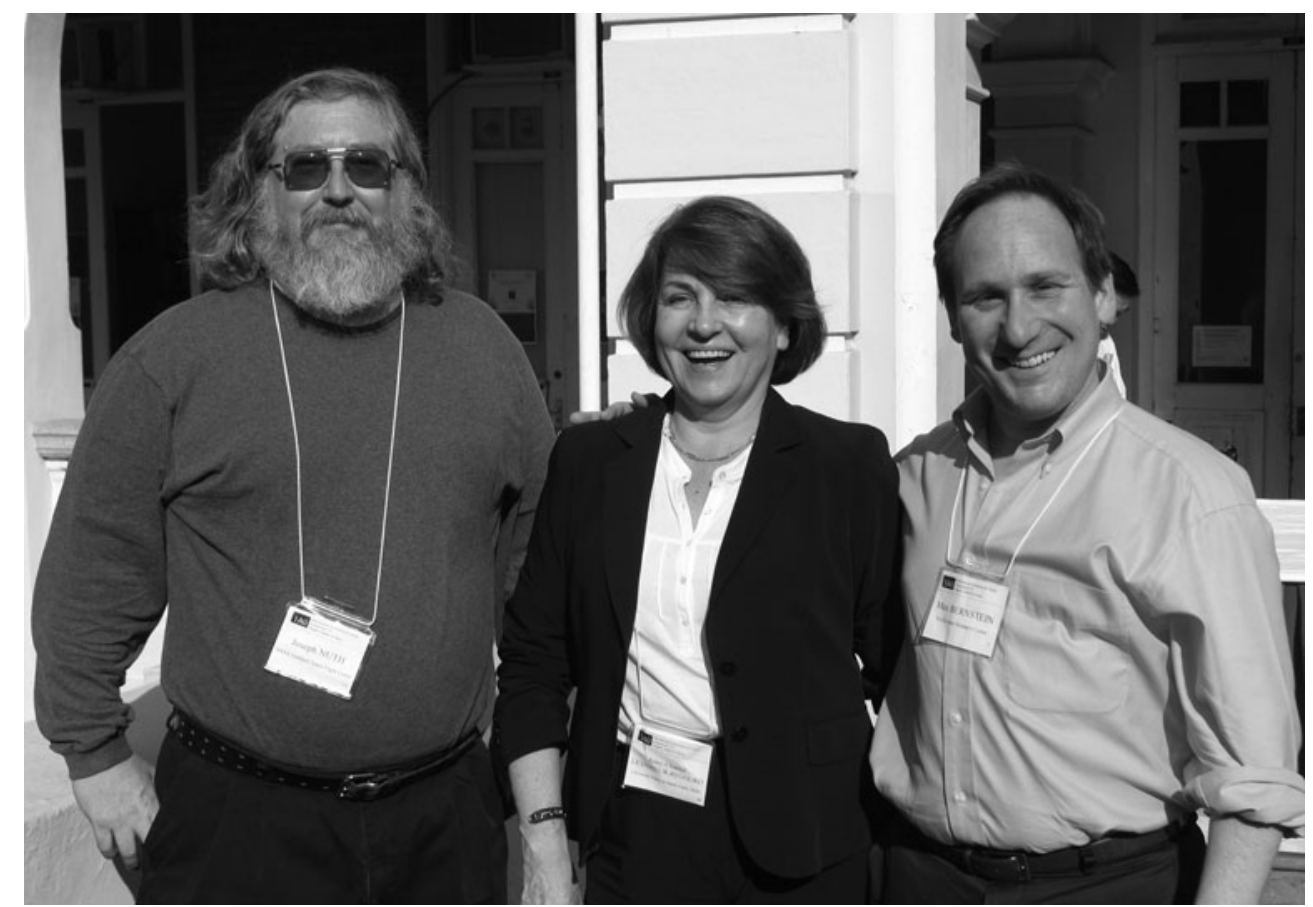

From left to right: Joe Nuth, Anny-Chantal Levasseur-Regourd, Max Bernstein (photo by Dale Cruikshank). 